\title{
Nephrotic Syndrome and a Retroperitoneal Mass: A Case Report of a Patient with Recurrent Invasive Thymoma
}

\author{
Hiroaki Myoga ${ }^{1}$, Tetsu Akimoto ${ }^{1}$, Naoko Mato ${ }^{2}$, Takakiyo Nakaya ${ }^{2}$, Takuya Murakami ${ }^{1}$, \\ Hiromichi Yoshizawa ${ }^{1}$, Saki Nakagawa ${ }^{1}$, Atsushi Miki ${ }^{1}$, Takahiro Masuda ${ }^{1}$, \\ Takahisa Kobayashi ${ }^{1}$, Yuko Ono ${ }^{3}$, Osamu Saito ${ }^{1}$, Yoshihiko Ueda ${ }^{3}$, \\ Shigeaki Muto ${ }^{1}$ and Daisuke Nagata ${ }^{1}$
}

\begin{abstract}
:
A 68-year-old man was admitted to our hospital to undergo an examination for nephrotic syndrome while concurrently complicated with recurrent thymoma in the parietal pleura and retroperitoneum. He had been diagnosed with invasive thymoma and had undergone thymo-thymectomy seven years previously. Based on the renal biopsy findings, his nephrotic syndrome was ascribed to minimal change disease. He was treated with corticosteroid monotherapy, which resulted in complete remission six months later, despite the fact that the recurrent thymoma remained. The role of thymoma in the pathogenesis of paraneoplastic glomerulopathy and the therapeutic concerns that emerged in this case are also discussed.
\end{abstract}

Key words: thymoma, paraneoplastic glomerulopathy, nephrotic syndrome, minimal change disease, corticosteroids

(Intern Med 56: 3317-3322, 2017)

(DOI: 10.2169/internalmedicine.9224-17)

\section{Introduction}

Nephrotic syndrome is a pivotal manifestation of glomerular injury associated with various kinds of neoplasms (1). Membranous nephropathy is a major kidney disease among nephrotic patients with solid malignancy, while other pathologies, such as minimal change disease (MCD), membranoproliferative glomerulonephritis, and focal segmental glomerulosclerosis can also manifest as paraneoplastic glomerulopathy $(2,3)$. Occasionally, such a relationship can be seen even in patients with thymoma, which is a common neoplasm of the anterior mediastinum arising from thymic epithelial cells (2-4). Nephrotic syndrome may either precede or act as the presenting feature of the disease, although there is often a substantial time interval between the 2 conditions, with renal manifestations occurring in approximately half of all cases 8 to 180 months after the curative treatment of thymoma, primarily based on surgical resection with radiotherapy (5-7).

In this report, we describe our experience with a case of nephrotic syndrome due to MCD, which is considered to be the most common type of Hodgkin's lymphoma-associated paraneoplastic glomerulopathy $(2,3)$, in a man with retroperitoneal recurrence of invasive thymoma.

\section{Case Report}

A 68-year-old man was admitted to our hospital in the beginning of July 2016 complaining of progressive swelling in his upper and lower limbs. Seven years earlier, he had been found to have asymptomatic microhematuria with a dysmorphic red blood cell predominance when he manifested a protracted cough and was diagnosed with invasive thymoma based on a systemic evaluation. He was then subjected to one session of neoadjuvant chemotherapy consist-

${ }^{1}$ Division of Nephrology, Department of Internal Medicine, Jichi Medical University, Japan, ${ }^{2}$ Division of Pulmonary Medicine, Department of Internal Medicine, Jichi Medical University, Japan and ${ }^{3}$ Department of Diagnostic Pathology, Dokkyo Medical University Koshigaya Hospital, Japan

Received: March 22, 2017; Accepted: April 24, 2017; Advance Publication by J-STAGE: October 11, 2017

Correspondence to Dr. Tetsu Akimoto, tetsu-a@jichi.ac.jp 

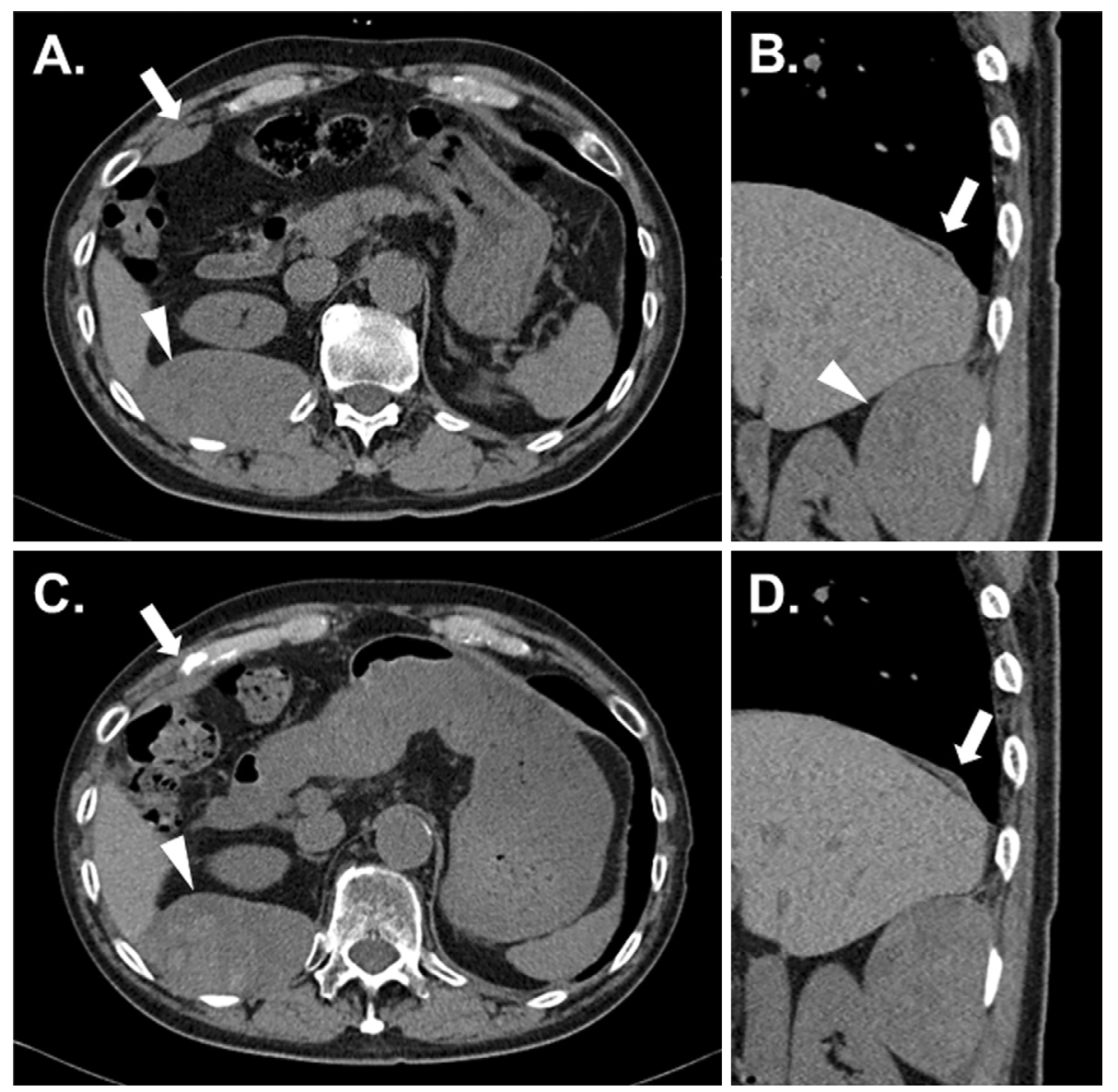

Figure 1. Radiographic findings. A select axial image of a CT examination performed in October 2015 (A) showed a right anterior pleural nodule (arrow) and a well-circumscribed mass (arrowhead) in the right retroperitoneal space, while a sagittal reconstructed image $(B)$ revealed the thickened parietal pleura (arrow) as well as the retroperitoneal mass (arrowhead). A select axial image of follow-up CT performed in August (C) revealed marginal changes in the thickness and size of the right retroperitoneal mass (arrowhead) despite a slight reduction in those of the right anterior pleural nodule (arrow). The appearance of the thickened parietal pleura (arrow) was also virtually unchanged (D).

ing of cisplatin, doxorubicin, vincristine, and cyclophosphamide with a marginal change in the overall size of the neoplastic lesion. Thereafter, right upper and middle pulmonary bilobectomy as well as thymo-thymectomy combined resection of the pericardium and right phrenic nerve was performed through median sternotomy, leading to a pathological diagnosis of type B3 disease according to the World Health Organization classification with Masaoka stage IIIA (8).

His postoperative recovery was uneventful, and a routine follow-up computed tomography (CT) examination performed at 67 years of age (October 2015) revealed a right anterior pleural nodule with a thickened pleura and a wellcircumscribed homogenous solid mass in the right retroperitoneal space without any remarkable symptoms (Fig. 1A and B). A CT-guided fine needle biopsy from the retroperitoneal mass lesion was diagnostic of metastatic thymoma, having a similar histological pattern to the primary neoplastic tissue. At the time, there were no signs of nephrotic syndrome, and his serum albumin (Alb) level was 4.1 $\mathrm{g} / \mathrm{dL}$, while a dipstick analysis revealed a $2+$ occult blood reaction despite a negative result for urine protein with a serum creatinine $(\mathrm{Cr})$ level of $0.95 \mathrm{mg} / \mathrm{dL}$. At the end of June 2016, his serum Cr level was $1.08 \mathrm{mg} / \mathrm{dL}$ when the patient started to complain about symptoms in his extremities. Seven days later, a laboratory analysis revealed a reduced serum Alb level of $1.9 \mathrm{~g} / \mathrm{dL}$, an increased serum $\mathrm{Cr}$ level of $2.29 \mathrm{mg} / \mathrm{dL}$, and heavy proteinuria of $21,400 \mathrm{mg} / \mathrm{gCr}$. The patient was therefore referred and admitted to our hospital for further work-up.

At admission (hospital day 1), he had gained approximately $7.5 \mathrm{~kg}$ in the previous 2 weeks, bringing his weight to $70.5 \mathrm{~kg}$. His blood pressure was $135 / 81 \mathrm{mmHg}$ with a pulse of 92 beats/min and temperature of $36.4^{\circ} \mathrm{C}$. No rashes or petechiae were observed. Renal sonography revealed that the right and left kidney measured $103 \times 44 \mathrm{~mm}$ and $102 \times 57$ $\mathrm{mm}$ in size, respectively, with normal renal cortex echogenicity. A laboratory evaluation revealed the following results: white blood cells, 5,500/ $\mu \mathrm{L}$; hemoglobin, $13.1 \mathrm{~g} / \mathrm{dL}$; platelet count, $20.4 \times 10^{4} / \mu \mathrm{L}$; fibrinogen, $568 \mathrm{mg} / \mathrm{dL}$, D- 

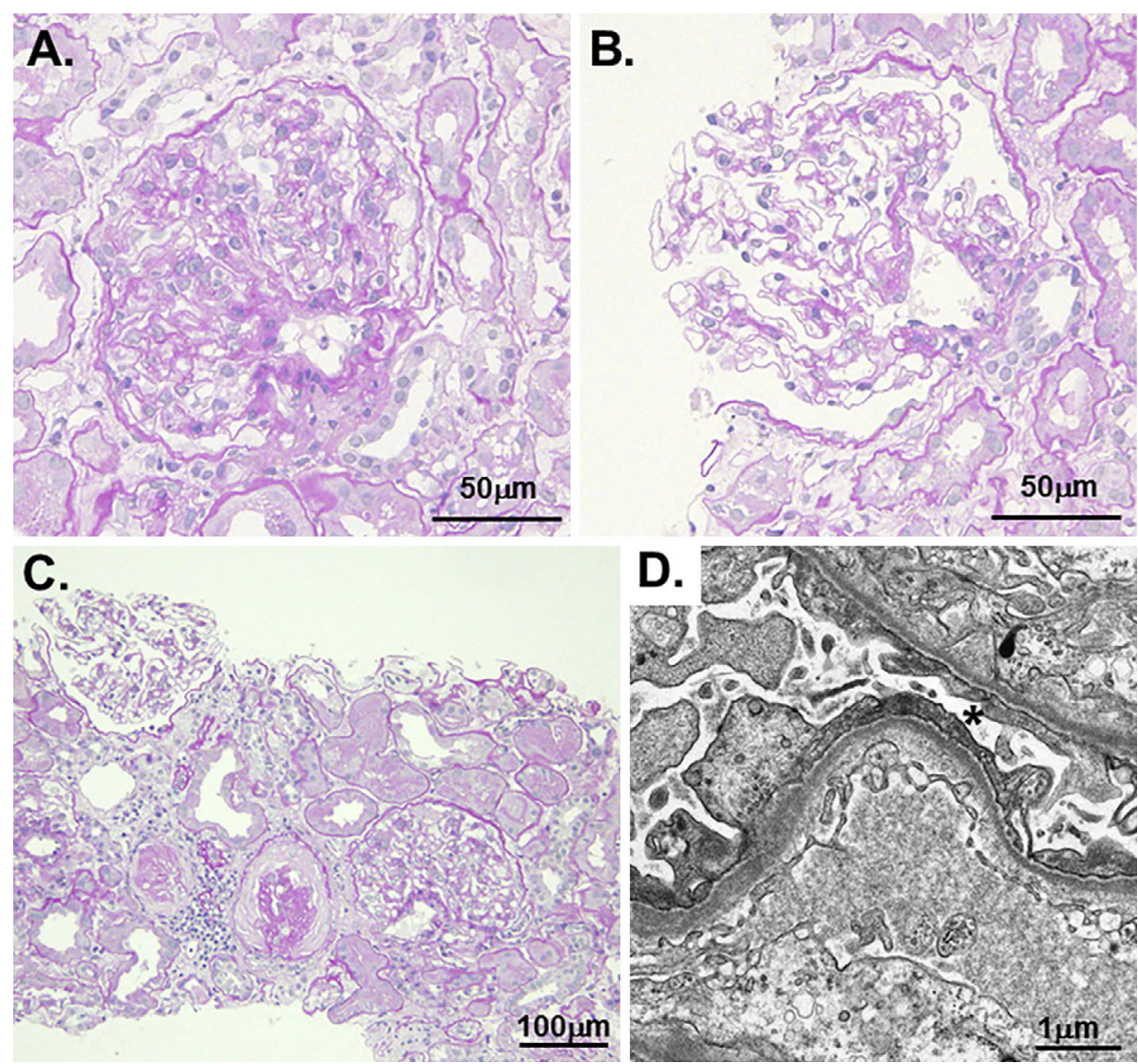

Figure 2. The renal biopsy findings. (A and B) Photomicrographs of the glomeruli in the different renal biopsy cores. Each light micrograph demonstrates a glomerulus showing minor abnormalities (Periodic acid-Schiff staining). (C) A low-power view indicates two glomeruli with a collapsed glomerular tuft with global wrinkling of the capillary walls and a urinary space filled with acellular material. The other glomeruli showed marginal glomerular changes (Periodic acid-Schiff staining). An electron micrograph of a portion of the glomerulus (B) demonstrates diffuse foot process effacement (asterisks). The scale bar is indicated in each panel.

dimer, $23.5 \mu \mathrm{g} / \mathrm{mL}$, blood urea nitrogen, $65 \mathrm{mg} / \mathrm{dL}$; serum $\mathrm{Cr}, 2.56 \mathrm{mg} / \mathrm{dL}$; total protein, $4.6 \mathrm{~g} / \mathrm{dL}$; serum Alb, $1.9 \mathrm{~g} /$ $\mathrm{dL}$; sodium, $143 \mathrm{mmol} / \mathrm{L}$; potassium, $4.5 \mathrm{mmol} / \mathrm{L}$; chloride $111 \mathrm{mmol} / \mathrm{L}$; calcium $7.6 \mathrm{mg} / \mathrm{dL}$; phosphorus $3.3 \mathrm{mg} / \mathrm{dL}$; asparate aminotransferase, $15 \mathrm{U} / \mathrm{L}$; alanine aminotransferase, $9 \mathrm{U} / \mathrm{L}$; fasting plasma glucose, $82 \mathrm{mg} / \mathrm{dL}$; hemoglobin A1c, $5.9 \%$; C-reactive protein, $0.19 \mathrm{mg} / \mathrm{dL}$; immunoglobulin (Ig) G, $776 \mathrm{mg} / \mathrm{dL}$; IgA, $170 \mathrm{mg} / \mathrm{dL}$; and IgM, $73 \mathrm{mg} / \mathrm{dL}$. He was positive for anti-nuclear antibodies, while tests for antineutrophil cytoplasmic antibodies, hepatitis B virus surface antigen, and antibodies to the hepatitis $\mathrm{C}$ virus were all negative. His urine was $3+$ for protein and contained $11.9 \mathrm{~g}$ of protein in a 24-h specimen, while the sediment showed 5 to 9 red blood cells per high-power field. The $\mathrm{Cr}$ clearance and proteinuria selectivity index were $36.3 \mathrm{~mL} / \mathrm{min}$ and 0.175 , respectively.

On hospital day 6, a renal biopsy revealed 3 cores of renal parenchyma with 35 glomeruli, almost half of which were globally sclerotic. The rest of the glomeruli showed an almost normal appearance, and there was marginal tubular atrophy. An immunohistochemical analysis failed to reveal any immune complex deposits, while electron microscopy showed flattening of the foot processes of the glomerular visceral epithelial cells without any apparent electron-dense deposits within the glomerular basement membrane or mesangial area, leading us to ascribe his nephrotic syndrome to MCD (Fig. 2).

Oral prednisolone (PSL) at a dose of $40 \mathrm{mg} /$ day was commenced on the day after the renal biopsy (hospital day 7). In addition, he was started on an increased dose of intravenous furosemide with a titrated dosage ranging from 300$700 \mathrm{mg} /$ day. Nevertheless, he remained grossly edematous, and the decline in his renal function steadily progressed, leading to an elevated serum $\mathrm{Cr}$ level of $4.28 \mathrm{mg} / \mathrm{dL}$ and a reduced urine volume of approximately $400 \mathrm{~mL}$ on hospital day 10. Thus, oral tolvaptan (15 mg/day) and spironolactone (50 mg/day) were added to the therapeutic regimen, and he was subjected to a transient hemodialysis (HD) program. He underwent a total of $6 \mathrm{HD}$ sessions between hospital days 10 and 24. During this time, his urine volume began to increase along with a gradual improvement of his edematous status. Another follow-up CT performed on hospital day 41 (mid-August 2016) revealed a marginal change in the thickness and size of the right retroperitoneal mass despite a 
slight reduction in those of the right anterior pleural nodule (Fig. 1C and D). Around the same time, diuretics were no longer required to control the patient's fluid status; however, he still had heavy proteinuria (3.6 g/day with an increased serum $\mathrm{Cr}$ level of $1.97 \mathrm{mg} / \mathrm{dL}$ ) on hospital day 39, despite the continued administration of oral PSL (40 mg/day). We therefore administered intravenous pulse therapy with methyl-PSL ( $500 \mathrm{mg} /$ day) for 3 consecutive days from hospital day 40 followed by oral PSL ( $35 \mathrm{mg} / \mathrm{day})$. At approximately 6 months after the renal biopsy, he was being treated with oral PSL (20 mg/day), having reached complete remission with a urine protein level of $100 \mathrm{mg} / \mathrm{gCr}$ despite the fact that the recurrent thymoma remained due to the conservative nature of its management, and his serum $\mathrm{Alb}$ and $\mathrm{Cr}$ levels settled around $3.8 \mathrm{~g} / \mathrm{dL}$ and $1.14 \mathrm{mg} / \mathrm{dL}$, respectively.

\section{Discussion}

The link between nephrotic syndrome and thymoma was first described over three decades ago by Posner et al. (9). Since then, several paraneoplastic kidney diseases have been identified among patients complicated by thymoma, with MCD being the most frequently reported glomerulopathy $(2,3)$. Numerous patients with thymoma-associated nephropathy may manifest renal insufficiency as well as nephrotic syndrome (6). In this regard, the clinical scenario of the current patient characterized by a set of events, including invasive thymoma, minimal change nephrotic syndrome, and elevations in serum Cr levels, which can be ascribed to acute kidney injury (AKI), may not be surprising. However, few systemic studies have been conducted on this topic $(5,6)$ due to the rarity of the disease. Indeed, only 2 cases with nephrotic syndrome were identified in a review of 960 cases of thymoma (10). We believe that the accumulation of more such cases will help clarify the nature of the disease.

The pathogenesis linking thymoma and MCD has yet to be delineated, but disturbances in the T-lymphocyte function resulting from a remote effect of thymectomy may induce nephrotic syndrome through the accelerated production of biological mediators (11), thereby increasing the glomerular permeability (6). The current case conflicts with this proposal in some ways, since our patient had already been complicated with recurrent thymoma at approximately 9 months before the onset of the symptoms of nephrotic syndrome. Information about the onset of nephrotic syndrome during the course of advanced thymoma is limited $(6,12-14)$, thus preventing us from evaluating the impact of this time lag on the overall disease process in the current patient. Nevertheless, we feel it is reasonable to consider that abnormalities in the immune system may result from thymoma as well as thymectomy, since the thymus is the primary stromal milieu guiding the maturation and selection of T-lymphocytes $(15,16)$. Interestingly, a detailed description of changes in the immune cell subset composition in recurrent thymoma-associated MCD was recently pub- lished (17), implying the presence of immunologic disturbance in subjects with recurrent disease. However, whether or not different histological types of thymoma result in distinct paraneoplastic glomerular injuries remains unclear. In the largest series of paraneoplastic glomerulopathies associated with thymoma, membranous nephropathy was exclusively identified in patients with epithelium-predominant type B3 thymoma; however, it has been shown in patients with other histologic types of thymoma as well $(5,9,18)$.

Steroid treatment appears to be well-tolerated and have acceptable benefits in select patients with thymomaassociated nephrotic syndrome due to MCD; however, previous studies demonstrating a good response to such a procedure are limited by their reliance on observational and retrospective data, making them vulnerable to confounding $(6,7)$. Adjunct therapy with cyclosporine, cyclophosphamide, and/or chemotherapeutic agents has also been practiced in some patients in remission for nephropathy $(14,19-21)$, but a lack of prospective data implies that the decisions made concerning therapy may be empirical. In the current case, the remnant state of the recurrent neoplasm as well as our failure to promptly perform tumor ablation with surgery, chemotherapy, and/or irradiation might have characterized the patient's overall response to the corticosteroids $(3,22,23)$, and this might lead to the delayed or incomplete recovery from the AKI despite our failure to confirm histological characteristics compatible with interstitial edema and ischemic tubular injury, which can be pathogenic bases for the disease among patients with $\operatorname{MCD}(24,25)$, in the renal biopsy specimens. Nevertheless, the lack of substantial information about the disease process of MCD accompanied by recurrent thymoma prevents us from objectively evaluating the validity of the overall outcome in the present patient. Why our patient had red blood cells in his urine throughout the observation period remains unclear; however, some patients may present with microscopic hematuria despite a normal ultrastructural appearance of the glomerular basement membrane (26), and it has been acknowledged that patients with MCD can occasionally manifest microscopic hematuria with a benign course (24-26).

Thymoma has an indolent oncological behavior. Recurrent disease is observed in 10-30\% of patients several years after radical surgery (27). This recurrence often follows a locoregional pattern, with the pleura and mediastinum being the most common sites (27); in contrast, there have only been a few anecdotal reports published describing the retroperitoneal recurrence of invasive thymoma (28-30). In the present patient, a histological evaluation was only performed for the retroperitoneal mass, preventing us from precisely evaluating the disease process of the recurrence. We also failed to confirm a distinct connection between the retroperitoneal lesion and the right pleural cavity in the diagnostic CT scan, as described previously $(28,29)$. Nevertheless, we feel it is reasonable to consider that the transdiaphragmatic spread originating from the pleural involvement might have played a 
role in our patient as well. Currently, treatment strategies for recurrent thymoma remain to be standardized, and no consensus regarding the optimum therapeutic option has yet been established, although surgical approaches have proven feasible with varying success in select patients $(27,31)$. Uneventful tumor resection has also been shown, even in patients with recurrent disease in the retroperitoneum (28-30). Alternatively, or in addition, there may be a subset of patients with recurrent thymoma who benefit from steroid treatment leading to tumor debulking $(32,33)$. Our failure to confirm a distinct benefit of the treatment with corticosteroids on the retroperitoneal mass may not be surprising, as type B3 thymoma has been shown to be somewhat resistant to such agents (33). When our patient started to manifest swelling in his upper and lower extremities, he did not agree to surgery promptly despite the absence of absolute contraindications; we therefore prioritized the diagnostic and therapeutic management for the patient's concurrent nephrotic syndrome. No clear recommendations regarding the appropriate timing for surgery have yet been made $(27,31)$; however, we believe that careful follow-up is mandatory in order to avoiding missing the opportunity to reintroduce a proposal for surgical intervention when applicable.

In conclusion, our case provides further indirect evidence that MCD may be a consequence of an imbalanced immune system resulting from acquired thymic disease. Monotherapeutic corticosteroids may be a therapeutic option for thymoma-associated MCD, although information regarding the appropriate dosage, optimal duration, and tapering protocol for these kinds of agents is lacking. Obviously, further experience from a larger number of cases similar to ours will be required to clarify the nature of the disease and establish optimum therapeutic strategies.

The authors state that they have no Conflict of Interest (COI).

\section{References}

1. Akimoto T, Otani N, Takeshima E, Saito O, Kusano E, Nagata D. Do we have to perform a renal biopsy? Clinical dilemmas in a case with nephrotic syndrome. Clin Med Insights Case Rep 7: 6770, 2014.

2. Bacchetta J, Juillard L, Cochat P, Droz JP. Paraneoplastic glomerular diseases and malignancies. Crit Rev Oncol Hematol 70: 39-58, 2009.

3. Lien YH, Lai LW. Pathogenesis, diagnosis and management of paraneoplastic glomerulonephritis. Nat Rev Nephrol 7: 85-95, 2011.

4. Thomas CR, Wright CD, Loehrer PJ. Thymoma: state of the art. J Clin Oncol 17: 2280-2289, 1999.

5. Schillinger F, Milcent T, Wolf C, Gulino R, Montagnac R. Nephrotic syndrome as the presenting feature of malignant thymoma. Nephrol Dial Transplant 12: 2732-2734, 1997.

6. Karras A, de Montpreville V, Fakhouri F, Grünfeld JP, Lesavre P; Groupe d'Etudes des Néphropathies Associées aux Thymomes. Renal and thymic pathology in thymoma-associated nephropathy: report of 21 cases and review of the literature. Nephrol Dial Transplant 20: 1075-1082, 2005.

7. Long Q, Wu P, Jiang G, Zhu C. Minimal change nephrotic syn- drome associated with invasive thymoma: a case report with literature review. Clin Nephrol 81: 296-301, 2014.

8. Wright CD. Management of thymomas. Crit Rev Oncol Hematol 65: 109-120, 2008.

9. Posner MR, Prout MN, Berk S. Thymoma and the nephrotic syndrome: a report of a case. Cancer 45: 387-391, 1980.

10. Rosenow EC 3rd, Hurley BT. Disorders of the thymus. A review. Arch Intern Med 144: 763-770, 1984.

11. Gerli R, Paganelli R, Cossarizza A, et al. Long-term immunologic effects of thymectomy in patients with myasthenia gravis. J Allergy Clin Immunol 103: 865-872, 1999.

12. Varsano S, Bruderman I, Bernheim JL, Rathaus M, Griffel B. Minimal-change nephropathy and malignant thymoma. Chest 77: 695-697, 1980

13. Lasseur $\mathrm{C}$, Combe $\mathrm{C}$, Deminière $\mathrm{C}$, Pellegrin JL, Aparicio $\mathrm{M}$. Thymoma associated with myasthenia gravis and minimal lesion nephrotic syndrome. Am J Kidney Dis 33: e4, 1999.

14. Teoh DC, El-Modir A. Managing a locally advanced malignant thymoma complicated by nephrotic syndrome: a case report. J Med Case Rep 2: 89, 2008.

15. Yamauchi J, Ubara Y, Suwabe T. Focal segmental glomerulosclerosis associated with invasive thymoma. Ther Apher Dial 15: 210211, 2011.

16. Takahama Y. Journey through the thymus: stromal guides for Tcell development and selection. Nat Rev Immunol 6: 127-135, 2006.

17. Gharwan H, Tomita Y, Lee MJ, et al. Alterations of immune cell subsets in relapsed, thymoma-associated minimal change disease: A case report. Oncol Lett 10: 1155-1158, 2015.

18. Valli G, Fogazzi GB, Cappellari A, Rivolta E. Glomerulonephritis associated with myasthenia gravis. Am J Kidney Dis 31: 350-355, 1998.

19. Ogawa M, Ueda S, Ohto M, et al. Minimal change nephrotic syndrome developed after non-surgical treatment of a thymoma. Clin Nephrol 38: 171-172, 1992.

20. Faur D, Martín N, Archuleta JM, Torguet P. Quiz page August 2012: a man with nephrotic syndrome and a mediastinal mass. Am J Kidney Dis 60: A25-A27, 2012.

21. Hsiao SY, Chang KY, Chang JY, Su WC. Nephrotic syndrome associated with thymoma. J Cancer Res Pract 1: 233-240, 2014.

22. Jayasena SD, Woolfson RG, Griffiths MH, Neild GH. Nephrotic syndrome, malignant thymoma, and myasthenia gravis. Case report and review of the literature. Am J Nephrol 15: 361-363, 1995.

23. Ishida I, Hirakata $H$, Kanai $H$, et al. Steroid-resistant nephrotic syndrome associated with malignant thymoma. Clin Nephrol 46: 340-346, 1996.

24. Mak SK, Short CD, Mallick NP. Long-term outcome of adultonset minimal-change nephropathy. Nephrol Dial Transplant 11: 2192-2201, 1996.

25. Waldman M, Crew RJ, Valeri A, et al. Adult minimal-change disease: clinical characteristics, treatment, and outcomes. Clin J Am Soc Nephrol 2: 445-453, 2007.

26. White RH. The investigation of haematuria. Arch Dis Child 64: 159-165, 1989.

27. Sandri A, Cusumano G, Lococo F, et al. Long-term results after treatment for recurrent thymoma: a multicenter analysis. J Thorac Oncol 9: 1796-1804, 2014.

28. Debnath J, Chawla N, Talwar R, et al. Pleural and transdiaphragmatic retroperitoneal metastasis developing two and half years after resection of invasive thymoma. Singapore Med J 49: e64-e67, 2008.

29. van Geffen WH, Sietsma J, Roelofs PM, Hiltermann TJ. A malignant retroperitoneal mass-a rare presentation of recurrent thymoma. BMJ Case Rep 2011 (Epub ahead of print).

30. Yang J, Li QQ, Ding YY, Cheng-de L. Recurrent thymoma in the 
retroperitoneal space: a rare case report. Clin Pract 5: 746, 2015.

31. Lucchi M, Mussi A. Surgical treatment of recurrent thymomas. J Thorac Oncol 5: S348-S351, 2010.

32. Tateyama H, Takahashi E, Saito Y, et al. Histopathologic changes of thymoma preoperatively treated with corticosteroids. Virchows Arch 438: 238-247, 2001.

33. Kobayashi Y, Fujii Y, Yano M, et al. Preoperative steroid pulse therapy for invasive thymoma: clinical experience and mechanism of action. Cancer 106: 1901-1907, 2006.

The Internal Medicine is an Open Access article distributed under the Creative Commons Attribution-NonCommercial-NoDerivatives 4.0 International License. To view the details of this license, please visit (https://creativecommons.org/licenses/ by-nc-nd/4.0/).

(C) 2017 The Japanese Society of Internal Medicine Intern Med 56: 3317-3322, 2017 